\title{
FORCED USE TREATMENT OF CHRONIC HEMIPARESIS
}

Twelve children (age 1 to 8 years) with chronic ( $>1$ year) hemiparesis were treated by forced use, or constraint-induced, movement therapy at Tulane University School of Medicine, New Orleans, LA. Each received a plaster cast on the unaffected arm for 1 month, while 13 hemiparetic control children did not. Peabody Developmental Motor Scales (PDMS) and parental reports, used to assess change in function, were obtained on all children at entry, and at 1 month, 6 months, and 7 months after entry. Controls received casts after 6 months.

The casted children improved 12.6 PDMS points after 1 month, while the controls showed only 2.5 points improvement. Improvements had persisted in 7 children who returned after:6 months for follow-up testing. Ten control children who received casts at crossoyer showed similar gains in function. The measured improvements were corroborated by parental reports. Ongoing physical/occupational therapy was received by both casted and control patients and did not explain the greater improvement observed in the casted group. (Willis JK, Morello A, Davie A et al. Forced use treatment of childhood hemiparesis. Pediatrics July 2002;110:94-96). (Reprints: Dr John K Willis, Department of Psychiatry and Neurology, HC82, Tulane University School of Medicine, 1430 Tulane Ave, New Orleans, LA 70112).

COMMENT. Constraint-induced movement therapy has been proven effective in the rehabilitation of adults with stroke and hemiparesis. The above study at Tulane has demonstrated the benefits of this technique in children with hemiparetic cerebral palsy. Cerebrocortical reorganization is postulated as the mechanism of this form of therapy.

\section{BRAIN NEOPLASMS}

\section{MANAGEMENT OF OPTIC CHIASMA/HYPOTHALAMIC GLIOMAS}

A retrospective chart review of all newly diagnosed tumors involving the optic chiasm from 1982-1996 was performed at British Columbia's Children's Hospital, Vancouver, Canada. Of 32 patients, 14 (10 male) had chiasmatic and 18 (9 male) chiasmatic/hypothalamic astrocytomas. Neurofibromatosis I was present in $10(71 \%)$ of the chiasmatic group and none of the chiasmatic/hypothalamic patients. Clinical presentations of chiasmatic tumors were decreased visual acuity (9), symptomatic neoplasms elsewhere (4), hydrocephalus (3), developmental delay (2), and precocious puberty (2). The most common presenting symptoms of chiasmatic/hypothalamic tumors were reduced visual acuity (11), elevated intracranial pressure (8), and diencephalic syndrome (7).

The majority (13) of chiasmatic tumors was managed by surveillance only, for an average follow-up of 5.7 years (range 1.9-14.8 yrs), and none showed progression requiring treatment. Six of the 14 had second non-optic gliomas ( 2 in the septum pellucidum, 2 brain stem gliomas, 1 temporo-patietal and 1 thalamic glioma) treated by resection, irradiation or chemotherapy.

The majority (17) of chiasmatic/hypothalamic tumors had surgical resection, subtotal in 8 , partial in 6 , limited in 3 ; one had no surgery. The extent of resection showed no correlation with the time to tumor progression (average 18 months). Limited resections were associated with fewer complications related to hypothalamic dysfunction. Diabetes insipidus developed in 5 of 8 with subtotal resection, all 6 with partial resection, and none with limited resection or biopsy only. The syndrome of inappropriate antidiuretic hormone secretion developed in 7 overall. One patient died in the early postoperative period and 4 died during 